\title{
Severe polyposis in $\mathrm{Apc}^{1322 \mathrm{~T}}$ mice is associated with submaximal Wnt signalling and increased expression of the stem cell marker Lgr5
}

\author{
Annabelle Lewis, ${ }^{1}$ Stefania Segditsas, ${ }^{1}$ Maesha Deheragoda, ${ }^{2,3}$ Patrick Pollard, ${ }^{4}$ \\ Rosemary Jeffery, ${ }^{3}$ Emma Nye, ${ }^{5}$ Helen Lockstone, ${ }^{6}$ Hayley Davis, ${ }^{1}$ Susan Clark, \\ Gordon Stamp, ${ }^{5}$ Richard Poulsom, ${ }^{3}$ Nicholas Wright, ${ }^{3}$ lan Tomlinson ${ }^{1}$
}

\begin{abstract}
- Additional figures and data are published online only. To view these files please visit the journal online (http://gut.bmj. com)
\end{abstract}

${ }^{1}$ Molecular and Population Genetics Laboratory, Wellcome Trust Centre for Human Genetics, University of Oxford, Oxford, UK

2Department of Pathology, University College Hospital, London, UK

${ }^{3}$ Histopathology Laboratory, London Research Institute Cancer Research UK, London, UK

${ }^{4}$ Oxygen Sensing Group, Henry Wellcome Building for Molecular Physiology, University of Oxford, Oxford, UK

${ }^{5}$ Experimental Histopathology Unit, London Research Institute, Cancer Research UK, London, UK

${ }^{6}$ Bioinformatics and Statistical Genetics, Wellcome Trust Centre for Human Genetics, University of Oxford, Oxford, UK ${ }^{7}$ The Polyposis Registry, St Mark's Hospital, Harrow, London, UK

\section{Correspondence to}

Dr Annabelle Lewis, Molecular and Population Genetics Laboratory, Wellcome Trust Centre for Human Genetics, University of Oxford, Oxford OX3 7BN, UK;

lewisa@well.ox.ac.uk

Revised 16 August 2010 Accepted 18 August 2010 Published Online First 6 October 2010

\section{ABSTRACT}

Background and aims Adenomatous polyposis coli $(A P C)$ is a tumour suppressor gene mutated in the germline of patients with familial adenomatous polyposis (FAP) and somatically in most colorectal cancers. APC mutations impair $\beta$-catenin degradation, resulting in increased Wnt signalling. The most frequent APC mutation is a codon 1309 truncation that is associated with severe FAP. A previous study compared two mouse models of intestinal tumorigenesis, Apc ${ }^{\text {R850X }}$ (Min) and $\mathrm{Apc}^{1322 \mathrm{~T}}$ (1322T), the latter a model of human codon 1309 changes. 1322T mice had more severe polyposis but, surprisingly, these tumours had lower levels of nuclear $\beta$-catenin than Min tumours. The consequences of these different $\beta$-catenin levels were investigated. Methods Enterocytes were isolated from 1322T and Min tumours by microdissection and gene expression profiling was performed. Differentially expressed Wnt targets and other stem cell markers were validated using quantitative PCR, in situ hybridisation and immunohistochemistry.

Results As expected, lower nuclear $\beta$-catenin levels in 1322T lesions were associated with generally lower levels of Wnt target expression. However, expression of the Wnt target and stem cell marker Lgr5 was significantly higher in 1322T tumours than in Min tumours. Other stem cell markers (Musashi1, Bmi1 and the Wnt target Cd44) were also at higher levels in 1322T tumours. In addition, expression of the Bmp antagonist Gremlin1 was higher in 1322T tumours, together with lower Bmp2 and Bmp4 expression.

Conclusions The severe phenotype caused by truncation of Apc at codon 1322 is associated with an increased number of stem cells. Thus, a submaximal level of Wnt signalling favours the stem cell phenotype and this may promote tumorigenesis. A level of Wnt signalling exists that is too high for optimal tumour growth.

\section{INTRODUCTION}

Adenomatous polyposis coli $(A P C)$ is a tumour suppressor gene that is mutated in patients with familial adenomatous polyposis and the majority of sporadic colorectal cancers. It is a component of the canonical Wnt signalling pathway, regulating the transcription factor $\beta$-catenin. Truncation or loss of APC disrupts the $\beta$-catenin degradation complex leading to an increase in nuclear $\beta$-catenin levels and aberrant expression of Wnt pathway target

\section{Significance of this study}

What is already known about this subject?

- Adenomatous polyposis coli (APC) is a tumour suppressor gene mutated in the germline of patients with familial adenomatous polyposis and somatically in most colorectal cancers. Apc mutations lead to increased levels of $\mathrm{Wnt}$ signalling.

- The 'just right' model proposes that different mutations in $A P C$ result in different levels of Wnt pathway activation and differing levels of tumour susceptibility, and that an intermediate level of Wnt signalling is optimal for the initiation of colorectal tumorigenesis.

- Two mouse models with different Apc mutations causing protein truncations at codon 850 (Min) and 1322 (1322T) show differences in the severity of intestinal polyposis, with the 1322 truncation causing more numerous and larger adenomas.

- This more severe phenotype is associated with a submaximal level of active/nuclear $\beta$-catenin.

What are the new findings?

- Lower nuclear $\beta$-catenin in 1322T adenomas is associated with mostly lower levels of Wnt target expression.

- Conversely, expression of the Wnt target and stem cell marker Lgr5 was significantly higher in 1322T tumours than in Min tumours, and in normal intestinal crypts from the former.

- Other stem cell markers (Musashi1, Bmi1 and the Wnt target $\mathrm{Cd} 44$ ) were also at higher levels in 1322T tumours.

How might it impact on clinical practice in the foreseeable future?

- Our findings suggest that higher levels of stem cells in both normal tissue and adenomas cause an increase in colorectal tumour numbers, size and dysplasia. Quantifying stem cells may help to predict the risk of colorectal tumours in humans.

- The 1322T mouse model of colorectal tumorigenesis has high numbers of stem cells and will make a useful system to test novel therapies directed specifically at cancer stem cells in the intestine.

- The confirmation of the 'just right' hypothesis provides valuable information about optimal levels of Wnt signalling in tumorigenesis and suggests that modulating Wnt levels either up or down may alter tumorigenesis. online under the BMJ Journals unlocked scheme, see http:// gut.bmi.com/site/about/ unlocked.xhtml 
genes. However, it has been difficult to determine which of the numerous Wnt target genes are primarily responsible for tumour initiation.

The 'just right' model ${ }^{12}$ proposes that different mutations in $A P C$ result in different levels of Wnt pathway activation and differing levels of tumour susceptibility. Few APC mutations found in vivo result in absence of protein function, and it is extremely rare to find a colorectal cancer in which neither mutant APC allele harbours a mutation after codon 1265. Germline mutations resulting in APC proteins truncated at codon 1309, for example, are relatively common and result in more severe familial adenomatous polyposis than proteins truncated at an earlier point. It is established that a 1309-truncated APC protein retains some $\beta$-catenin binding and degradation activity-for example, through the 20 -amino acid $\beta$-catenin binding repeat (20AAR) that it retains-whereas shorter proteins do not. ${ }^{3} 4$ These findings suggest that an intermediate level of Wnt signalling is optimal for the initiation of colorectal tumorigenesis.

We recently tested this hypothesis by performing a detailed phenotypic and molecular comparison of two Apc-mutant mice on the C57BL6/J background: the $A p c^{R 850 X}$ (Min) mouse model which is heterozygous for a truncating mutation at codon 850 and the $A p c^{1322 T}$ (1322T) model which is heterozygous for an allele functionally equivalent to the human 1309 mutation. ${ }^{4}$ In both animals, tumours usually develop when the wild-type allele undergoes loss of heterozygosity by mitotic recombination. We found that the $1322 \mathrm{~T}$ mouse did indeed develop more severe polyposis, with more tumours developing at a faster rate than in the Min model. The 1322T tumours, however, had much lower levels of nuclear $\beta$-catenin.

The main physiological difference between the 1322T and Min intestines was the increased incidence of crypt fission in normal and polyp tissue from the former. Crypt fission has been proposed as a mechanism of early tumorigenesis ${ }^{5}$ and may therefore underlie the more rapid adenoma development seen in the $1322 \mathrm{~T}$ mice. It has been proposed ${ }^{7}$ that crypt fission occurs when stem cell numbers in a crypt exceed some threshold level. In early colorectal adenomas it is plausible that the same model applies, and that tumorigenesis is driven by increased numbers of stem-like cells, leading to crypt fission and hence increased adenoma size and number.

It has previously been difficult to test the connection between crypt fission, stem cells and tumorigenesis due to the lack of accurate intestinal stem cell markers. However, recently there has been a number of papers identifying putative stem cell markers in the gastrointestinal tract. One of the most compelling of these markers is Lgr5 (leucine-rich-repeat-containing Gprotein-coupled receptor 5), also known as Gpr49, a Wnt target gene. ${ }^{8}$ Lineage tracing experiments have shown that Lgr5-positive crypt base columnar (CBC) cells contribute to all epithelial lineages in the intestine and that specific mutation of $A p c$ in Lgr5-positive CBC cells efficiently drives adenoma formation. ${ }^{9}$ Indeed, single Lgr5-positive cells can generate crypt-like structures in vitro in specific culture conditions. ${ }^{10}$ The function of the Lgr5 protein itself, however, is not well understood in the intestine and Lgr 5 knockout mice die at neonatal stages due to a tongue defect. Therefore it is likely that Lgr5 is purely a marker that is co-expressed with unknown genes that confer the intestinal stem cell phenotype. ${ }^{11}$

Another intestinal stem cell marker verified using lineage tracing experiments is the polycomb ring finger protein, Bmil. ${ }^{12}$ There are some differences between the Bmi1- and Lgr5-positive cell populations, with Bmil being found predominantly at the
+4 crypt position rather than in CBCs and only towards the proximal end of the small intestine. This raises the possibility of more than one stem cell population existing in the small intestine, although the different populations might overlap. The neural RNA-binding protein Musashi1 (Msi1) is also found specifically in the +4 cells but has not been investigated in great detail. ${ }^{13}{ }^{14}$ CD 44, like Lgr5, is a Wnt target gene and has been proposed as an intestinal stem cell marker due to the capacity of expressing cells to propagate tumours as xenografts. ${ }^{15}{ }^{16}$ Prominin1 (CD133), which has also been shown to mark tumorigenic cells in xenograft studies, is expressed in the same CBC cells as Lgr5, but is also present in transit amplifying cells further up the crypt, suggesting that it is not a strict stem cell marker. ${ }^{17-20}$

In this study we have used expression profiling to gain clues about the important differences in Wnt target expression between tumours from $1322 \mathrm{~T}$ and Min mice. In addition, we have used multiple markers to examine the hypothesis that the more severe tumorigenesis in 1322T than Min mice is driven by higher numbers of stem-like cells in the former. The conclusions support the notion that maintenance of a stem-like phenotype in adenoma cells promotes tumorigenesis.

\section{METHODS}

\section{Mouse breeding and collection of samples}

All 1322T and Min animals were housed in the Barrier Unit of Cancer Research UK, Clare Hall Laboratories and all work was approved by the UK Home Office and local ethics committee. $1322 \mathrm{~T}$ or Min mice were sacrificed at a specified age or when symptomatic (anaemic secondary to polyps or suffering rectal prolapse). The intestinal tract was divided into four segments, consisting of stomach, three equal lengths of small intestine (proximal (SB1), middle (SB2), and distal (SB3)), and large intestine. Each segment was flushed with phosphate buffered saline (PBS), opened longitudinally and laid out on filter paper. The samples were fixed in $10 \%$ neutral buffered formalin for $3 \mathrm{~h}$ and stored in $70 \%$ ethanol or embedded in OCT (Sigma, St Louis, MO, USA) and snap frozen in liquid nitrogen.

\section{Laser capture microdissection, extraction of RNA and microarray analysis}

Nine serial $8 \mu \mathrm{m}$ cryosections were taken from OCT-embedded intestine preparations. Every third section was stained with $\mathrm{H} \& \mathrm{E}$ using standard protocols. The remaining sections were incubated in ice-cold $70 \%$ ethanol for $2 \mathrm{~min}$, cresyl violet staining solution ( $1 \%$ cresyl violet acetate, $50 \%$ ethanol) for $30 \mathrm{~s}$ and washed briefly in $70 \%$ ethanol followed by $100 \%$ ethanol. After air drying for 1-2 min, laser capture microdissection was carried out using the PALM system. Cell types were distinguished in the serial H\&E sections and areas of the adenomas containing enterocytes with no Paneth cells were selected. The same area was captured from 5-6 sections for each tumour and the RNA was extracted using the PicoPure RNA extraction kit (Applied Biosystems, Life Technologies, Carlsbad, California, USA) and treated with RNase-free DNase I (Qiagen, Hilden, Germany). RNA quality was assessed on a Bioanalyzer (Agilent, Santa Clara, CA, USA). Three 1322T and three Min tumour samples were sent to the Paterson Institute Microarray Service where they were hybridised to GeneChip Mouse Exon 1.0 ST Arrays (Affymetrix, Santa Clara, CA, USA). Output Cel files were analysed using the $\mathrm{R}$ statistical programme with the robust multichip average (RMA) method, which is implemented in the BioConductor package 'Affy'. Differentially expressed genes were then identified using the linear models of microarray analysis (LIMMA) package. 


\section{Array validation by quantitative PCR (Q-PCR)}

RNA was extracted from microdissected adenoma tissue as above and cDNA was synthesised using the Stratagene Affinity script Q-PCR kit (Applied Biosystems, Life Technologies, Carlsbad, CA, USA). Q-PCR was carried out on a 7900 Fast Real-time PCR machine using pre-optimised Taqman probes and primers (Applied Biosystems), and normalised to Gapdh. Analysis was carried out using the standard $\Delta \Delta \mathrm{Ct}$ method.

\section{In situ hybridisation}

mRNA in situ hybridisation (ISH) was carried out using $4 \mu \mathrm{m}$ serial sections from formalin-fixed paraffin-embedded mouse intestines. Riboprobes were generated for each gene of interest. Specifically for the Lgr 5 probe, a 562 bp long PCR product was generated from mouse tumour cDNA, spanning from the $5^{\prime}$ UTR to exon 5 (primers were $5^{\prime}$-CTGCTCTCTGCTCCCAGTCT-3' and $5^{\prime}$-GGACGTCTGTGAGAGCAT- $3^{\prime}$ ) and cloned into pGem3Z. For CD44, a 446 bp long PCR product spanning the final four exons (which are common in all transcripts) was generated from mouse tumour cDNA, (primers were $5^{\prime}$ GGCTCCTTACCAGGAGATCG-3' and 5'-CGTAGGCACTACACCCCAAT-3'). For hybridisation control, a $\beta$-actin probe (414 bases) was used. Riboprobes were generated by in vitro transcription using SP6 polymerase and labelled with ${ }^{35}$ S-UTP or ${ }^{3} \mathrm{H}$-UTP (GE Healthcare, Chalfont St Giles, UK). Other methods were as described by Poulsom et al. ${ }^{21}$ Signal intensity for assays using ${ }^{35}$ S-UTP labelled probes was scored in at least 10 polyps from each type of mouse (by SS and AL) blinded to polyp origin, as long as the $\beta$-actin signal passed a quality control threshold. Total expression was calculated as the proportion of the epithelial component of a polyp that expressed the mRNA of interest. This was done by capturing simultaneous bright field and dark field images for each adenoma and using the ImageJ software package to measure the total area of the epithelial component of the adenoma and the area showing mRNA expression. A ratio was calculated from these areas to give the proportion of expressing cells. For Lgr5, where mRNA expression intensity differed between $1322 \mathrm{~T}$ and Min lesions, that proportion was multiplied by the estimated mean intensity of the test mRNA measured on a semiquantitative scale from 0 to 3 (absent $=0$, weak $=1$, moderate $=2$, strong $=3$ ) compared with $\beta$-actin control and with reference to surrounding normal tissue. In ${ }^{3} \mathrm{H}$-UTP-based assays 135 normal crypts of each genotype were scored for the number of positive cells ( $\geq 4$ silver granules present).

\section{Immunohistochemistry}

Immunohistochemistry was carried out using $4 \mu \mathrm{m}$ sections from the same formalin-fixed paraffin-embedded tissues used for ISH. Sections were dewaxed, hydrated, blocked with hydrogen peroxide and antigen retrieval was carried out by microwaving in sodium citrate buffer for $10 \mathrm{~min}$. Normal goat serum diluted to $1: 1250$ in $1 \%$ BSA was used as a blocking agent for $30 \mathrm{~min}$. The following primary antibodies were added for 1-4 h: polyclonal rabbit anti-Bmi1 antibody (Abgent, AP2513b) diluted to 1:150 or polyclonal rabbit anti-musashi antibody (Chemicon, AB5977) 1:200. Bmi1 stained sections were washed in PBS before applying a secondary antibody (biotinylated goat anti-rabbit DAKO, 1:1250) for $1 \mathrm{~h}$ at room temperature. Sections were washed in PBS and incubated in ABC (Vector Laboratories, Burlingham, CA, USA) for 30 min before washing. 3,3'-diaminobenzidine (DAB) solution (Sigma) was applied for $2-5 \mathrm{~min}$ and development of the colour reaction was monitored microscopically. Slides were washed in PBS, stained with a light haematoxylin, dehydrated, cleared and then mounted using Din-butyl phthalate in Xylene (DPX). Msi1 stained sections were incubated with secondary antibody (molecular probes goat antirabbit Alexa 488, 1:300 dilution) for $1 \mathrm{~h}$, immersed in Sudan black $(0.1 \%$ in $70 \%$ IMS) to reduce the autofluorescence of paraffin sections and mounted in hard set VECTAMOUNT (Vector Laboratories) with 4', 6-diamino-2-phenylindole (DAPI). Positive cells were scored as present or absent in at least 10 polyps from each type of mouse by $\mathrm{MD}$ while blinded to polyp origin.

\section{Statistical analysis}

Kruskal-Wallis and Wilcoxon non-parametric tests were carried out using the STATA 9.0 package, Student t tests were carried out in Excel and the microarray analysis was carried out using R, as described above.

\section{RESULTS}

Our previous findings showed that adenoma numbers and size were greater in mice carrying a $1322 \mathrm{~T}$ mutation than in those with a Min mutation. This differential phenotype has remained stable in 1322T animals from 10 to $>20$ generations of backcrossing with $\mathrm{C} 57 \mathrm{Bl} / 6 \mathrm{~J}$. We have confirmed it is due to the location of the Apc truncating mutation by intercrossing the two strains and showing segregation of the more severe phenotype with the 1322T genotype. Polyp numbers were significantly higher and polyps larger in the F1 1322T mice than in the Min. Overall, however, the numbers of polyps caused by both 1322T and Min mutations fell after the intercross, suggesting selection for recessive positive modifiers and consistent with informal observations that inbreeding increases the severity of both the 1322T and Min phenotype.

Levels of nuclear $\beta$-catenin and active $\beta$-catenin were consistently lower in $1322 \mathrm{~T}$ than in Min adenomas. ${ }^{4}$ We wondered whether this would result in lower levels of Wnt target gene expression in the former. We therefore carried out mRNA expression profiling using exon microarrays in enterocytes from three Min and three $1322 \mathrm{~T}$ adenomas of similar size from the SB2 region of the small intestine. Since $1322 \mathrm{~T}$ tumours contain high frequencies of Paneth cells, which appear to be terminally differentiated and hence not to be progenitors of tumour growth, we used laser capture microdissection to exclude this lineage from the analysis. Figure $1 \mathrm{~A}$ shows the Wnt pathway target genes (http://www.stanford.edu/ rnusse/pathways/ targets.html) that are differentially expressed (fold change $>1.3$ ) between the two strains. As expected, there are more Wnt targets upregulated in Min than in 1322T adenomas $(p=0.0003$, sign test; see table 1 in the online supplement for results from all Wnt target genes, figure $1 \mathrm{~A}$ in the online supplement for expression levels of differentially expressed genes and figure 2 in the online supplement for further analyses of Wnt pathway and target genes).

Selected significantly different genes were then validated using quantitative RT-PCR (Q-PCR) on both the original samples and on a further independent microdissected sample set of five $1322 \mathrm{~T}$ and four Min tumours. Two of the more highly expressed Wnt target genes with altered expression could be validated ( $p<0.05$, Student t test): Lgr 5 , a known intestinal stem cell marker, which was upregulated by nearly threefold in 1322 T adenomas and wnt inhibitory factor 1 (Wif1) which was increased by nearly fourfold in Min adenomas (figure 1B). Axin2 and $T c f 7$ were more highly expressed in Min samples, but not significantly. Other putative Wnt target genes from the Bmp pathway (Bmp2, Bmp4, and Grem1) showed significant 

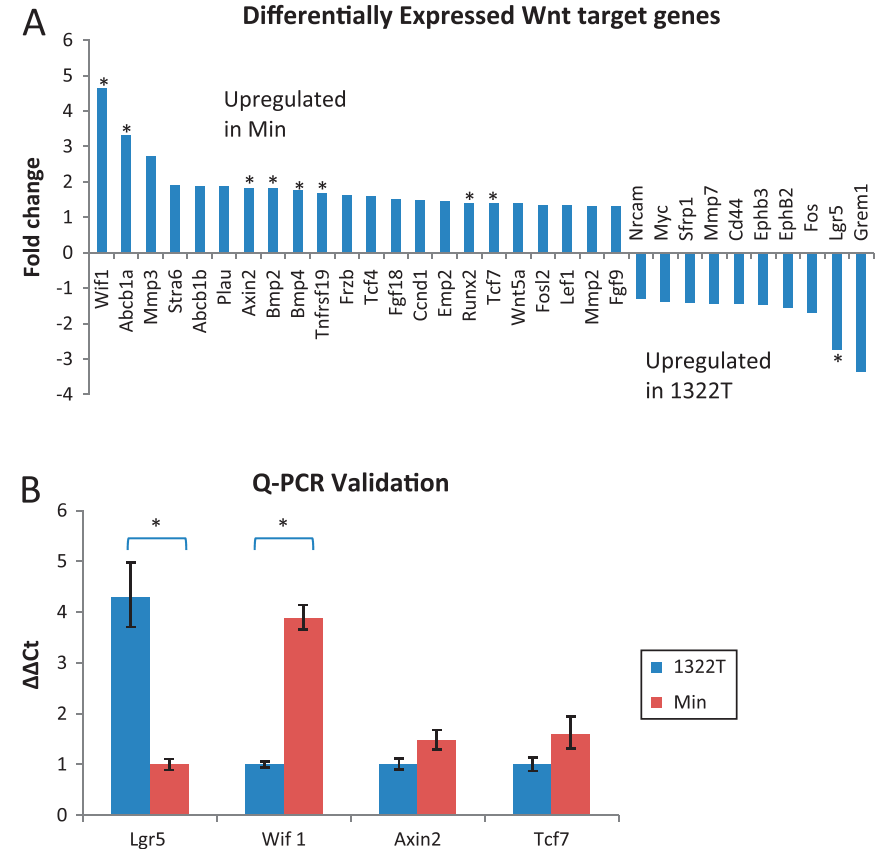

Figure 1 Differential expression of Wnt target genes. RNA extracted from laser captured microdissected tissue from three 1322T and three Min adenomas was analysed using exon arrays (Affymetrix) and differentially expressed Wnt targets were determined using the $R$ statistical package with Bioconductor and LIMMA tools. (A) Graph showing fold changes of differentially expressed Wnt targets (fold change $>1.3$ ) with genes upregulated in Min shown above the $x$-axis and $1322 T$ below. Highly significant differential genes $(p<0.01)$ are marked with an asterix. (B) Graph showing differentially expressed genes validated by $0-P C R$ analysis $\left({ }^{*} p<0.05\right)$. The housekeeping gene Gapdh was used as a normalisation control and the fold change was calculated using the $\Delta \Delta \mathrm{Ct}$ method.

expression changes in our microarray, with Q-PCR analysis demonstrating changes in the same direction to the arrays in all cases although these were not formally significant (see figure $1 \mathrm{~B}$ in online supplement). Q-PCR analysis is often unable to reliably detect small (less than twofold) differences, especially when there is some biological variability. Given the importance of the Bmp pathway in stem cell regulation and colorectal cancer, ${ }^{22} 23$ and the fact that three genes in this pathway show changes, these small differences in expression could be biologically relevant and should not be discounted.

We were particularly interested in the fact that $\operatorname{Lg} r 5$ expression was increased in 1322T tumours, because of its role as a marker of intestinal stem cells and because the lower nuclear $\beta$ catenin levels had led us to expect that Wnt target gene expression would be lower in 1322T tissue. We therefore decided to investigate the distribution of Lgr5 expression in more detail using isotopic ISH on sections of intestinal adenoma. We found that Lgr 5 expression occurred in large patches in these tumours, and that the patches were larger and more intense in 1322T tumours than Min lesions of similar size (figure 2A,B, intensity $\times$ area, $p=0.0008$; intensity, $p=0.004$; area, $p=0.065$; Wilcoxon test). In general, Lgr5 expression in tumours was much more extensive than in normal tissue where it was confined to the bottom of the crypts, as previously described. ${ }^{8}$

We then determined whether stem cell numbers, as detected by Lgr5 expression, might also differ between 1322T and Min mice in normal tissues on the basis that this might play a causative role in early microadenoma formation. Using
${ }^{3} \mathrm{H}$-labelled riboprobes to provide high resolution (figure $2 \mathrm{C}$ ), positive cells (defined as those with $\geq 4$ signals) were counted in normal crypts in 1322T, Min and wild-type samples. Only fulllength longitudinally orientated crypts with identifiable cell types $>10$ crypts away from tumour tissue were included in the study. The high resolution and sensitivity of this assay meant that we were able to detect Lgr 5 expression in most crypts and confirm that Lgr5-positive cells are found in the base of the crypt, usually in the $\mathrm{CBC}$ position. A small but significant $(p=0.002$, Kruskal-Wallis test) difference between strains was detected, with an increased number of Lgr5-positive cells occurring in 1322T but not in Min crypts compared with wildtype mice (figure 2D). An additional benefit of the high resolution ISH was that we were able to confirm that the large majority of Paneth cells in both 1322T and Min tumours and normal tissues were not positive for Lgr 5 expression, ruling out the possibility that a change in cellular composition was the reason for the high levels of Lgr5 detected by standard ISH in $1322 \mathrm{~T}$ tumours (see figure 3 in online supplement).

These data, together with our observation of an increase in crypt fission in $1322 \mathrm{~T}$ tumours, ${ }^{4}$ suggested that a reason for the more severe phenotype in 1322T mice was an increased number of stem cells in both normal tissues and early tumours. To provide further evidence for this hypothesis, we carried out analyses of other intestinal stem cell markers by immunohistochemistry (IHC) or ISH. For Bmil we found only a small number of cells which showed expression in normal tissue, many fewer than we identified in the Lgr 5 ISH experiments and too few to conduct a meaningful analysis in normal crypts. However, in tumours there were significantly more Bmi1-positive putative stem cells in 1322T than Min adenomas $(p=0.0001$, Wilcoxon test, figure 3A). Msi1 expression was observed in a similar number of adenoma cells to Bmi1 and was again significantly more prevalent in $1322 \mathrm{~T}$ tumours $(p=0.0011$, Wilcoxon test, figure $3 \mathrm{~B}$ ). Cd44, also a putative stem cell marker for mouse tissue (figure $3 \mathrm{C}$ ). Expression of $\mathrm{Cd} 44$ was much more widespread in tumours than Bmi1 and Msi1 and was found in the same cell populations as Lgr5 as well as additional areas, staining virtually all cells in $1322 \mathrm{~T}$ tumours but with a more patchy pattern in Min tumours $(\mathrm{p}=0.0212)$. Q-PCR analysis confirmed the upregulation of $C d 44$ in $1322 \mathrm{~T}$ tumours (see figure 1 in online supplement).

Since all the stem cell markers we investigated showed an increase in 1322T tumours, we assessed whether the increase in stem-like cells was consistent with the faster growth of adenomas seen in $1322 \mathrm{~T}$ mice. To do this, we carried out mathematical modelling using our model based on tumorigenesis by failure of differentiation of stem cells (see figure 4 in online supplement). ${ }^{24}$ We mimicked the differences between $1322 \mathrm{~T}$ and Min animals by assuming that the probability of a stem cell undergoing differentiation was slightly lower on the $1322 \mathrm{~T}$ than the Min genetic background. Even small differences in this probability gave profound differences in the rate of cell expansion and thus could account for the differences between the $1322 \mathrm{~T}$ and Min phenotypes.

\section{DISCUSSION}

Adenomas in $A p c^{1322 T}$ mutant mice are larger, more numerous and of earlier onset than those from $A p c^{M i n}$ animals in which the mutant protein is truncated at codon 850 and lacks all $\beta$-catenin binding repeats. We have previously shown that adenomas from 1322T mice have lower nuclear $\beta$-catenin than Min adenomas. Here we found, as predicted, that lower levels of $\beta$-catenin are 
Figure 2 1322T normal and adenoma tissue contains more Lgr5-positive cells. Lgr5 ISH was carried out on $4 \mu \mathrm{m}$ sections of formalin-fixed paraffinembedded 1322T and Min intestines.

(A) Bright field images $(10 \times$ objective magnification) of tumours showing patches of $L g r 5$ expression (visualised by silver granules) and counterstained with Giemsa. The signal is more widespread and more intense in the 1322T adenoma. (B) Box and whiskers diagram showing increased $\operatorname{Lgr} 5$ staining scored in 1322T and Min polyps ( $n=10$ for each genotype). For each polyp the area of staining was calculated as a proportion of the whole epithelial component and multiplied by the estimated mean intensity of the test mRNA. 1322T adenomas were scored much higher than Min $(p=0.0008$, Wilcoxon test). (C) Bright field images of in situ hybridisation carried out using a ${ }^{3} \mathrm{H}$-labelled $\operatorname{Lgr} 5$ probe at $60 \times$ magnification showing normal crypt tissue. Positive cells are outlined. Enlargements of selected cells are shown in the bottom panel. More expression can be seen in 1322T crypts than in Min and wild-type crypts. (D) Box and whiskers plot showing that more Lgr5-positive cells were present in 1322T crypts than in Min and wild-type normal crypts $(\mathrm{p}=0.002$,

Kruskal-Wallis test); 135 crypts of each genotype were scored for the number of positive cells ( $\geq 4$ silver granules present). In most cases
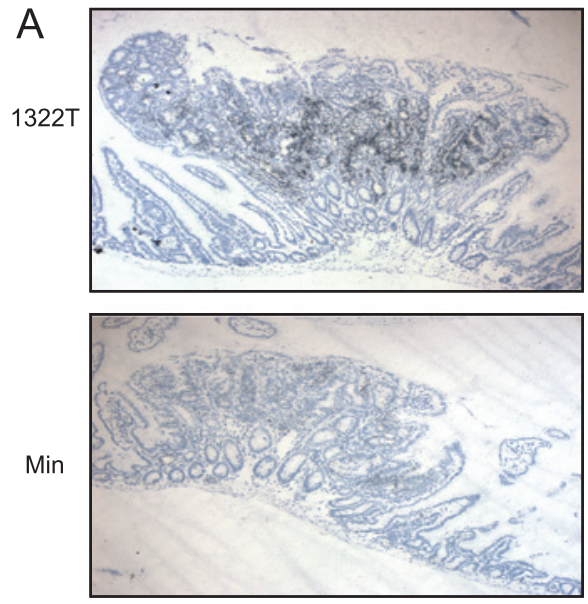

C
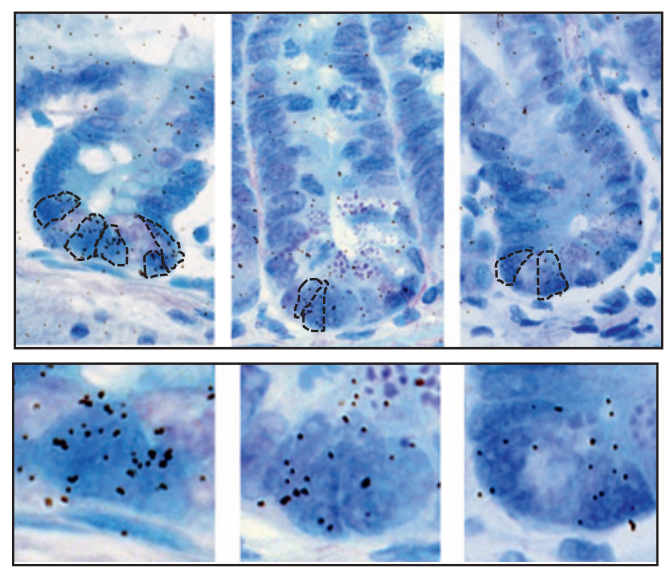

1322

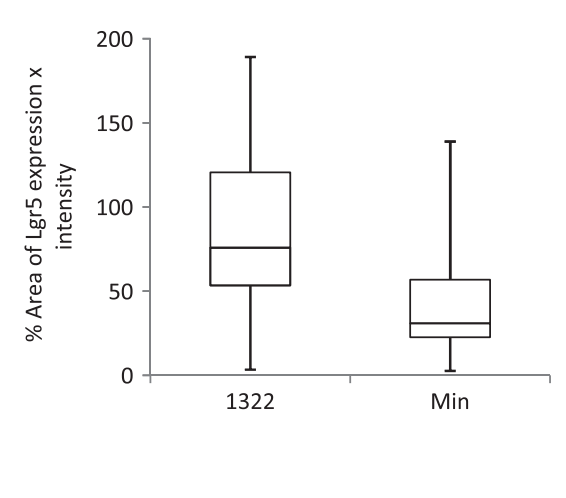

B

D

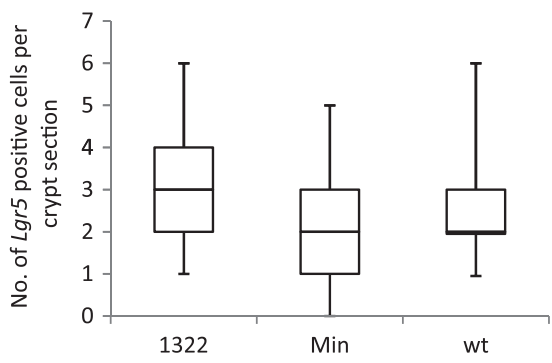

accompanied by generally lower expression of Wnt pathway genes, including the Wnt inhibitory factor Wif1. However, stem cell markers-including both Wnt targets such as Lgr5 and Cd44 and non-Wnt targets such as Bmi1 and Msi1-are upregulated in 1322T tumours compared with Min tumours. This suggests that the levels of Wnt signalling resulting from the homozygous 1322 T mutation found in tumours are closer to the optimum for promoting expression of genes that cause the stem cell phenotype than are the Wnt levels found in Min tumours. The stem cell phenotype is thus favoured by submaximal Wnt signalling.

We observed subtle differences in the numbers of cells expressing Lgr5 in normal tissue prior to tumour initiation, suggesting that haploinsufficiency of Apc has a detectable effect on stem cell numbers. This effect may explain some of the differences in tumour initiation if, according to the cancer stem cell model of tumorigenesis, the tumour-causing 'second hit' first occurs in a stem cell and then spreads by clonal expansion. However, a much larger variation in stem cell number occurs in tumours of the same size and location between mouse strains than can be explained by this effect alone. Tumour progression must therefore differ as well, and cells which already have a second hit at codon 1322 favour Lgr5 expression and stem cell characteristics.

More than half of the epithelial portion of the $1322 \mathrm{~T}$ adenomas showed Lgr5 and/or C 444 expression which could indicate that all these cells are (cancer) stem cells. While this is possible, it is more likely that these cells have some stem cell-like characteristics such as the ability to self-replicate, or that these cells have the potential to be stem cells given the right local conditions or niche. It is notable that Bmil and Msi1 protein expression was detected in far fewer cells overall. While some of these differences may be explained by technical factors such as threshold levels of detection for ISH and IHC as well as differences between the levels and stability of the respective mRNAs and proteins, it may be that there are different and dynamic populations of stem-like cells that express different markers at different points in time. We must also consider that the presence of stem cell markers does not necessarily mean that all the cells they mark function as stem cells or cancer stem cells in vivo. There is currently no straightforward functional assay that identifies stem cells, but lineage tracing experiments in reporter mice-possibly in conjunction with allograft studies using tumour stem cells from 1322T and Min animals-could be used in the future to identify which marker or marker combination most accurately defines the stem cell population.

Although high levels of expression of the target gene $\mathrm{LgrS}$ indicate increased stem cells in $1322 \mathrm{~T}$ adenomas, the functional role of the Lgr5 protein itself still remains unclear. It is therefore likely that other primary or secondary Wnt targets are important. Another Wnt pathway gene, Wif1, was differentially expressed, with higher levels in Min tumours. While not previously known to play a major role in colorectal cancer, Wif1 expression has been 
Figure 3 Stem cell markers are generally increased in 1322T adenomas. (A) Immunohistochemistry carried out with anti-Bmi1 antibody on 1322T and Min adenomas shown at $20 \times$ objective magnification. Box and whiskers diagram showing more Bmi1-positive cells were present in 1322T adenomas than in Min adenomas $(n=14$ for each genotype, median 7.5/1000 compared with 2.5/1000 in Min polyps; $p=0.0001$, Wilcoxon test). (B) Fluorescent immunohistochemistry carried out with anti-Msi1 antibody on 1322T and Min adenomas shown at $20 \times$ objective magnification. Box and whiskers diagram showing more Msi1-positive cells were present in 1322T adenomas than in Min adenomas $(n=10$ for each genotype, median of 8.5 cells/1000 counted in the $1322 \mathrm{~T}$ adenomas compared with 2.5 cells/1000 in Min adenomas matched for location and size; $p=0.0011$, Wilcoxon test). (C) Isotopic in situ hybridisation carried out with CD44 riboprobe on 1322T and Min adenomas shown at $20 \times$ objective magnification in dark field. Silver granules stain areas of CD44 expression. Box and whiskers diagram showing a larger area staining positive for CD44 in 1322T adenomas than in Min adenomas $(\mathrm{n}=13$ for $1322 \mathrm{~T}$ and $\mathrm{n}=9$ for Min, median area of $44 \%$ showing staining in 1322T adenomas compared with $35 \%$ in Min adenomas). For each polyp the area of staining was calculated as a proportion of the whole epithelial component using ImageJ software.

A Bmi1

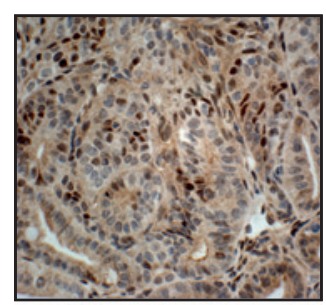

$1322 T$

B Msi1

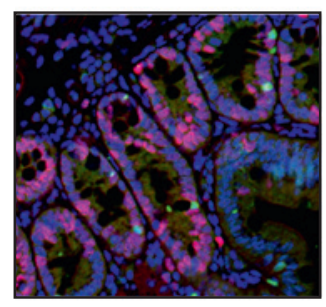

$1322 \mathrm{~T}$

C $\mathrm{Cd} 44$

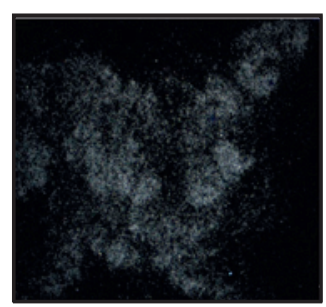

$1322 \mathrm{~T}$

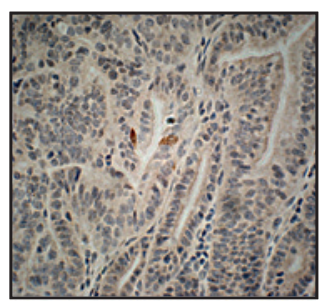

Min

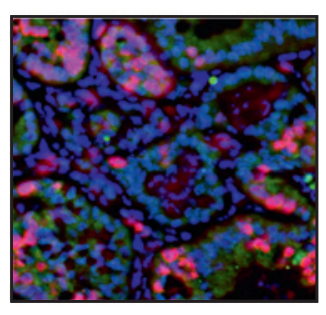

Min

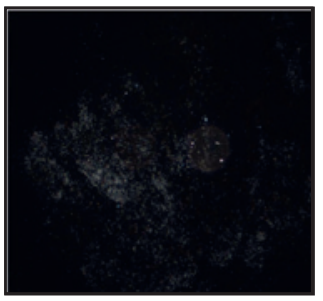

Min
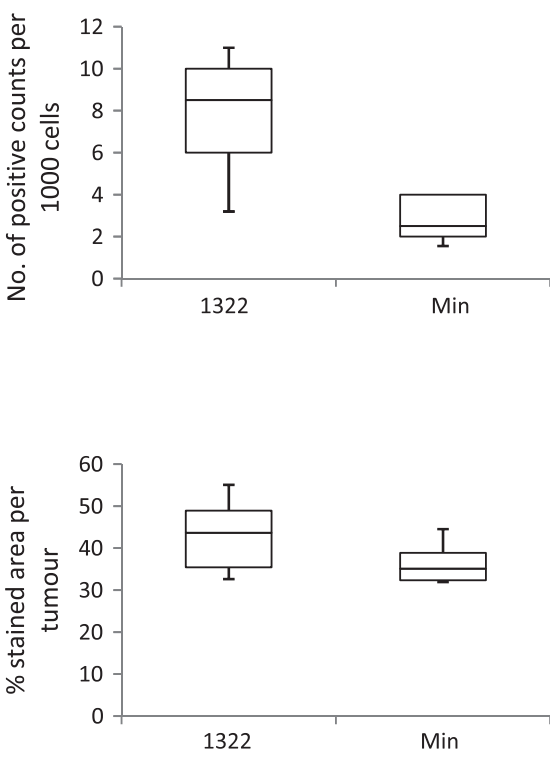

shown to inhibit bladder tumour growth and osteosarcomas and is often silenced by methylation in breast carcinomas and colorectal cancer cell lines. ${ }^{25-28}$ Wif1 is unlikely to be a direct Wnt target gene, but it has been proposed that it acts as part of a negative feedback loop. ${ }^{29}$ Wif1 expression could potentially play a role in the Min adenomas, repressing tumour growth-perhaps by repressing the stem cell phenotype-despite high levels of nuclear $\beta$ catenin and Wnt signalling. In support of this, Wif1 expression is generally low or absent in familial adenomatous polyposis tumours. ${ }^{30}$ Since it is counterintuitive that the feedback effect of Wif1 is stronger than the Wnt signal, it is necessary to postulate additional factors such as selective downstream effects for Wif1 to play a role in causing the phenotypic differences between the 1322T and Min animals.

Our expression arrays showed a small increase in expression of several other Wnt targets in 1322T adenomas including EphB2, EphB3, Grem1, Mmp7 and Myc. Unlike Lgr5, these increases were not validated by Q-PCR, possibly due to the large variability between tumours, but they may still give us further insights into the mechanisms leading to tumorigenesis or increased stem cell number. The Grem 1 gene is normally expressed in subepithelial myofibroblasts and found only at low levels in adenomas. Interestingly, however, it is an inhibitor of TGF $\beta / \mathrm{Bmp}$ signalling, a pathway that is proposed to inhibit stem cell renewal, ${ }^{31}$ so higher levels of Grem1 expression, together with the lower levels of Bmp2, Bmp4 and Bmp5 expression seen in 1322T tumours, could contribute to the observed stem cell phenotype.
In addition, expression of the matrix metalloproteinase $M M P 7$ correlates with poor prognosis in patients with colorectal cancer and promotes tumorigenesis in Min mice. ${ }^{32-34}$ It is also expressed highly in Paneth cells which are over-represented in 1322T adenomas. Since Paneth cells lie within the normal intestinal stem cell compartment, it is possible that MMP7 also influences stem cell numbers. Similarly, the difference in $M y c$ expression, although small, could play an important role in disease severity. Myc is essential for the phenotype caused by $A p c$ mutation in the intestine, and many of the genes upregulated after Apc loss, including the EphB receptors, are dependent on Myc expression. ${ }^{35}$ It is more difficult to explain the role of EphB in the severe $1322 \mathrm{~T}$ phenotype. EphB signalling is important for cell compartmentalisation and migration in the intestine, ${ }^{36}$ but it is thought that high levels limit the expansion of adenomas. ${ }^{37}$ However, the expression of the EphB genes is highest at the base of intestinal crypts in normal tissue, with $E p h B 3$ restricted exclusively to the region containing the stem cells, which could explain the high levels found in the stem cellrich $1322 \mathrm{~T}$ tumours.

Our use of mouse models in this study has allowed us to analyse in detail the effect of the location of specific germline and somatic Apc mutations to test the 'just right' hypothesis. Both our strains were kept on identical genetic backgrounds and no confounding mutations were seen at the loci. In addition, we have excluded the existence of unlinked modifiers in our mouse models by intercross experiments. While it is difficult to 
completely eliminate the possibility of tightly-linked modifiers in cis, an independent targeting event resulted in the same phenotype as our $1322 \mathrm{~T}$ model, ${ }^{4}$ suggesting that the targeted mutation alone is responsible for the severe polyposis and characteristic distribution of adenomas.

Confirmation of these results in samples from human patients has, however, proved difficult. As a result of the observations in human familial adenomatous polyposis adenomas which led to this hypothesis-namely, that a germline mutation early in APC is very rarely accompanied by an early somatic mutation-suitable samples to carry out analyses on patients with familial adenomatous polyposis are not available. In general, however, increased Lgr 5 expression is seen in all familial adenomatous polyposis adenomas and the levels of nuclear $\beta$-catenin are very low (see figures 5 and 6 in online supplement), comparable to $1322 \mathrm{~T}$ adenomas and consistent with the 'just right' hypothesis.

Clearly, many questions remain about how the expression of Wnt targets depends on nuclear $\beta$-catenin levels and other factors in both normal and tumour tissues. Some Wnt target genes known to be involved in intestinal tumorigenesis showed no significant difference between our two mouse models. For instance, no change was seen in Tcf1 expression which has been shown to suppress tumorigenesis in mammary tissue and also in the intestines of Min mice. ${ }^{38}$ We suggest that simple linear dependence of target levels is unlikely, and that different targets respond differently to changes in Wnt signalling. Our data confirm the operation of the 'just right' model of intestinal tumorigenesis $^{12}$ and suggest that it acts through promoting the stem cell phenotype, although the underlying causes for the strong selective constraints on APC mutations in colorectal cancer are likely to be complex.

Acknowledgements We are grateful to colleagues from the Biological Resources Unit and Histopathology Unit, London Research Institute, Cancer Research UK and to the Paterson Institute Microarray Facility. We thank E Kyriakides for help with samples for microdissection and, T Guenther and the St Marks Polyposis Registry for help with identification of FAP patient samples.

Funding Cancer Research UK, London; Biomedical Research Centre, Oxford, UK.

Competing interests None.

Ethics approval This study was conducted with the approval of the UK Home Office and the Oxfordshire research ethics committee.

Provenance and peer review Not commissioned; externally peer reviewed.

\section{REFERENCES}

1. Lamlum $\mathbf{H}$, llyas $\mathbf{M}$, Rowan $A$, et al. The type of somatic mutation at APC in familial adenomatous polyposis is determined by the site of the germline mutation: a new facet to Knudson's 'two-hit' hypothesis. Nat Med 1999;5:1071-5.

2. Albuquerque C, Breukel $\mathrm{C}$, van der Luijt $\mathrm{R}$, et al. The 'just-right' signaling model: APC somatic mutations are selected based on a specific level of activation of the beta-catenin signaling cascade. Hum Mol Genet 2002;11:1549-60.

3. Schneikert J, Grohmann A, Behrens J. Truncated APC regulates the transcriptional activity of beta-catenin in a cell cycle dependent manner. Hum Mol Genet 2007:16:199-209.

4. Pollard P, Deheragoda M, Segditsas S, et al. The Apc 1322T mouse develops severe polyposis associated with submaximal nuclear beta-catenin expression. Gastroenterology 2009:136:2204-13.

5. Leedham SJ, Thliveris AT, Halberg RB, et al. Gastrointestinal stem cells and cancer: bridging the molecular gap. Stem Cell Rev 2005;1:233-41.

6. Preston SL, Wong WM, Chan A0, et al. Bottom-up histogenesis of colorectal adenomas: origin in the monocryptal adenoma and initial expansion by crypt fission. Cancer Res 2003;63:3819-25.
7. Loeffler M, Bratke T, Paulus U, et al. Clonality and life cycles of intestinal crypts explained by a state dependent stochastic model of epithelial stem cell organization. $J$ Theor Biol 1997:186:41-54

8. Barker N, van Es JH, Kuipers J, et al. Identification of stem cells in small intestine and colon by marker gene Lgr5. Nature 2007;449:1003-7.

9. Barker N, Ridgway RA, van Es JH, et al. Crypt stem cells as the cells-of-origin of intestinal cancer. Nature 2009;457:608-11.

10. Sato T, Vries RG, Snippert HJ, et al. Single Lgr5 stem cells build crypt-villus structures in vitro without a mesenchymal niche. Nature 2009:459:262-5.

11. Morita H, Mazerbourg S, Bouley DM, et al. Neonatal lethality of LGR5 null mice is associated with ankyloglossia and gastrointestinal distension. Mol Cell Biol 2004:24:9736-43.

12. Sangiorgi E, Capecchi MR. Bmi1 is expressed in vivo in intestinal stem cells. Nat Genet 2008:40:915-20.

13. Kayahara T, Sawada M, Takaishi S, et al. Candidate markers for stem and early progenitor cells, Musashi-1 and Hes1, are expressed in crypt base columnar cells of mouse small intestine. FEBS Lett 2003;535:131-5.

14. Potten CS, Booth C, Tudor GL, et al. Identification of a putative intestinal stem cell and early lineage marker; musashi-1. Differentiation 2003;71:28-41.

15. Dalerba P, Dylla SJ, Park IK, et al. Phenotypic characterization of human colorecta cancer stem cells. Proc Natl Acad Sci USA 2007;104:10158-63.

16. Wielenga VJ, Smits R, Korinek V, et al. Expression of CD44 in Apc and Tcf mutant mice implies regulation by the WNT pathway. Am J Pathol 1999:154:515-23.

17. O'Brien CA, Pollett A, Gallinger $S$, et al. A human colon cancer cell capable of initiating tumour growth in immunodeficient mice. Nature 2007:445:106-10.

18. Ricci-Vitiani L, Lombardi DG, Pilozzi $\mathrm{E}$, et al. Identification and expansion of human colon-cancer-initiating cells. Nature 2007:445:111-15.

19. Snippert HJ, van Es JH, van den Born M etal. Prominin-1/CD133 marks stem cells and early progenitors in mouse small intestine. Gastroenterology 2009;136:2187-94.e1.

20. Zhu L, Gibson P, Currle DS, et al. Prominin 1 marks intestinal stem cells that are susceptible to neoplastic transformation. Nature 2009:457:603-7.

21. Poulsom R, Longcroft JM, Jeffery RE, et al. A robust method for isotopic riboprobe in situ hybridisation to localise mRNAs in routine pathology specimens. Eur $\mathrm{J}$ Histochem 1998:42:121-32.

22. Houlston RS, Webb E, Broderick $P$, et al. Meta-analysis of genome-wide association data identifies four new susceptibility loci for colorectal cancer. Nat Genet 2008:40:1426-35

23. Hardwick JC, Kodach LL, Offerhaus GJ, et al. Bone morphogenetic protein signalling in colorectal cancer. Nat Rev Cancer 2008;8:806-12.

24. Tomlinson IP, Bodmer WF. Failure of programmed cell death and differentiation as causes of tumors: some simple mathematical models. Proc Natl Acad Sci USA 1995;92:11130-4.

25. Kansara M, Tsang M, Kodjabachian L, et al. Wnt inhibitory factor 1 is epigenetically silenced in human osteosarcoma, and targeted disruption accelerates osteosarcomagenesis in mice. J Clin Invest 2009:119:837-51.

26. Tang Y, Simoneau AR, Liao WX, et al. WIF1, a Wnt pathway inhibitor, regulates SKP2 and c-myc expression leading to G1 arrest and growth inhibition of human invasive urinary bladder cancer cells. Mol Cancer Ther 2009;8:458-68.

27. Taniguchi $\mathbf{H}$, Yamamoto $\mathrm{H}$, Hirata $\mathrm{T}$, et al. Frequent epigenetic inactivation of Wnt inhibitory factor-1 in human gastrointestinal cancers. Oncogene 2005:24:7946-52.

28. Veeck J, Wild PJ, Fuchs T, et al. Prognostic relevance of Wnt-inhibitory factor-1 (WIF1) and Dickkopf-3 (DKK3) promoter methylation in human breast cancer. BMC Cancer 2009;9:217.

29. Reguart N, He B, Xu Z, et al. Cloning and characterization of the promoter of human Wnt inhibitory factor-1. Biochem Biophys Res Commun 2004;323:229-34.

30. Segditsas S, Sieber 0, Deheragoda M, et al. Putative direct and indirect Wnt targets identified through consistent gene expression changes in APC-mutant intestinal adenomas from humans and mice. Hum Mol Genet 2008;17:3864-75.

31. He XC, Zhang J, Tong WG, et al. BMP signaling inhibits intestinal stem cell self-renewal through suppression of Wnt-beta-catenin signaling. Nat Genet 2004;36:1117-21.

32. Adachi $\mathbf{Y}$, Itoh $\mathrm{F}$, Yamamoto $\mathrm{H}$, et al. Matrix metalloproteinase matrilysin (MMP-7) participates in the progression of human gastric and esophageal cancers. Int $\mathrm{J}$ Oncol 1998;13:1031-5.

33. Adachi $\mathbf{Y}$, Yamamoto $\mathrm{H}$, Itoh $\mathrm{F}$, et al. Contribution of matrilysin (MMP-7) to the metastatic pathway of human colorectal cancers. Gut 1999;45:252-8.

34. Wilson CL, Heppner KJ, Labosky PA, et al. Intestinal tumorigenesis is suppressed in mice lacking the metalloproteinase matrilysin. Proc Natl Acad Sci USA 1997:94:1402-7

35. Sansom 0J, Meniel VS, Muncan V, et al. Myc deletion rescues Apc deficiency in the small intestine. Nature 2007;446:676-9.

36. Merlos-Suarez A, Batlle E. Eph-ephrin signalling in adult tissues and cancer. Curr Opin Cell Biol 2008:20:194-200.

37. Cortina C, Palomo-Ponce S, Iglesias M, et al. EphB-ephrin-B interactions suppress colorectal cancer progression by compartmentalizing tumor cells. Nat Genet 2007:39:1376-83.

38. Roose J, Huls G, van Beest M, et al. Synergy between tumor suppressor APC and the beta-catenin-Tcf4 target Tcf1. Science 1999:285:1923-6. 